\title{
Differences in the strength of distractor inhibition do not affect distractor-response bindings
}

\author{
Carina Giesen • Christian Frings $\cdot$ Klaus Rothermund
}

Published online: 12 November 2011

(C) Psychonomic Society, Inc. 2011

\begin{abstract}
Distractor inhibition and distractor-response binding were investigated in two experiments by analyzing distractor repetition benefits and their interaction with response repetition effects in a sequential-priming paradigm. Distractor repetition benefits were larger for distractors that were incompatible with the to-be-executed response (task-related distractors) than for distractors that were not assigned to a response (neutral distractors), indicating that the strength of distractor inhibition was a function of response interference for the distractors. In contrast, the distractor-response bindings were found to be of equal strength for both task-related and neutral distractors. Thus, differences in the strengths of distractor inhibition did not affect the integration of distractors with responses into event files. Instead, our results suggest that distractor-response binding and distractor inhibition are independent mechanisms that are recruited for the automatization of behavior and action control.
\end{abstract}

Keywords Event files · Inhibition · Stimulus-response binding $\cdot$ Repetition priming $\cdot$ Episodic retrieval $\cdot$ Selective attention

In order to maneuver our way through the endless stream of information to perceive, offering infinite

\footnotetext{
C. Giesen $\cdot$ K. Rothermund $(\bowtie)$

Department of Psychology, Friedrich-Schiller-Universität Jena, Am Steiger 3, Haus 1,

D-07743 Jena, Germany

e-mail: klaus.rothermund@uni-jena.de

C. Frings

Saarland Universität,

Saarbrücken, Germany
}

possibilities to behave in the world around us, our brain has to rely on a limited number of simple and efficient processes and mechanisms. One important mechanism is selective attention - that is, the ability to discriminate between relevant and irrelevant information. Attending and responding to only those aspects of our environment that are related to our goals, and not being distracted by stimuli that are irrelevant for or might interfere with the current task, is important for the topdown control of behavior (Tipper, 1992). Selective attention is commonly assumed to entail two components (Houghton \& Tipper, 1994): Preferred processing of relevant information (activation) is accompanied by an active suppression of irrelevant, distracting information (inhibition). That is, once a stimulus is identified as being irrelevant, inhibition dampens the activation of the distractor representation (Houghton \& Tipper, 1994) or blocks its access to the response system to reduce interference (Fuentes, Vivas, \& Humphreys, 1999; Tipper \& Cranston, 1985).

Yet not each and every aspect of our behavior can be intentionally controlled. Other mechanisms are therefore necessary that translate intentional actions, resulting from a controlled and resource-demanding processing of information, into efficient behavioral routines and habits. The retrieval of previous behavioral episodes plays an important role for the automatization of behavior: According to recent instance-based models, a specific stimulus can become integrated with a response that is executed in close temporal proximity to the occurrence of the stimulus. The compound of stimulus and response is then stored as an instance (Logan, 1988) or event file (Hommel, 1998, 2004) in episodic memory. Reencountering the stimulus of such an event file leads to retrieval of the entire episode from memory, including the 
associated response (e.g., Denkinger \& Koutstaal, 2009; Hommel, 1998, 2004; Logan, 1988; Pösse, Waszak, \& Hommel, 2006). This retrieval of previous actions operates fast and automatically, exerting efficient bottom-up control of behavior by establishing stimulus-driven behavioral routines.

Recent evidence has suggested that the storage and retrieval of event files is not restricted to relevant stimulus features. Even distractors can be integrated with responses, so that subsequent presentations of a distractor stimulus can also lead to retrieval of the response that was executed during a previous encounter with the distractor (Rothermund, Wentura, \& De Houwer, 2005; see also Akçay \& Hazeltine, 2007; Frings, Rothermund, \& Wentura, 2007; Gibbons \& Stahl, 2008; Mayr \& Buchner, 2006; Mayr, Buchner, \& Dentale, 2009). These findings suggest that attending to targets apparently does not prevent an integration of distractors into event files.

The aim of the present research is to more closely investigate the interplay between these two mechanisms (inhibition of distractors and integration of distractors into event files) in selection situations. In particular, we compared distractor-response bindings and inhibition processes for response-incompatible and neutral distractors. Neutral distractors are not associated with a specific response; that is, they are irrelevant with respect to the current task. Response-incompatible distractors, on the other hand, are associated with a response that is part of the current task set. Incompatible distractors compete with the relevant target stimulus during response selection, which is why they produce stronger interference than do neutral distractors (Eriksen \& Eriksen, 1974; Wühr \& Müsseler, 2005).

The distinction between incompatible and neutral distractors implicates different cognitive processing demands. Shielding the response selection process against misspecifications requires that inhibition should primarily target response-incompatible distractors, because only these distractors have the potential to trigger an incorrect response. In line with this prediction, stronger inhibition effects were found if distractors exhibited features that were relevant (as compared to irrelevant) for the to-be-performed task (De Houwer, Rothermund, \& Wentura, 2001; Frings \& Wentura, 2006; Levinthal \& Lleras, 2008; Tipper, Weaver, \& Houghton, 1994). Similarly, some findings have suggested that the strength of distractor inhibition increases if selection of the target stimulus becomes more difficult, indicating that inhibition is activated specifically in response to those distractor stimuli that compete or conflict with the target (Fox, 1994; Grison \& Strayer, 2001; Houghton, Tipper, Weaver, \& Shore, 1996; Stablum, Ricci, Pavese, \& Umiltà, 2001).
Provided that the relative amounts of inhibition targeted at incompatible and neutral distractors differ, this might indirectly affect the creation and retrieval of distractor-response bindings. A major focus of our research thus consisted of comparing the storage and retrieval of distractor-response bindings for incompatible and neutral distractors. A straightforward hypothesis would state that the binding of incompatible distractors with responses is impeded or prevented because incompatible distractors are inhibited, and thus are excluded from binding processes and event files (Loach, Botella, Privado, \& Tsotsos, 2008; Loach \& Marí-Beffa, 2003), whereas neutral distractors are not inhibited or are inhibited to a lesser degree, and thus can become included in event files. Thus, if inhibition and distractor-response binding interact, we should observe (stronger) inhibition effects for incompatible distractor and (stronger) distractor-response bindings for neutral distractors.

However, inhibition has recently been shown to operate in a highly specific manner, targeting only those properties of a distractor stimulus that directly compete with the target for the control of action (Tipper, 2001; Tipper et al., 1994; see also De Houwer et al., 2001; Frings \& Wentura, 2006; Levinthal \& Lleras, 2008). More specifically, the main function of inhibition may be to prevent the access of incompatible distractors to response selection processes (Tipper \& Cranston, 1985; see also Fuentes et al., 1999; Neill, 2007). This blocking of distractor-response relations does not necessarily prevent the processing of the distractor at a perceptual or conceptual level, and it might also be compatible with an integration of distractors into event files. Such a specific and flexible view of the distractor inhibition mechanism would suggest that the processes of distractor-response binding and distractor inhibition operate more or less independently of each other (Mayr et al., 2009), allowing for an integration of neutral as well as of inhibited incompatible distractors into event files.

Investigating whether inhibition does or does not influence the binding of a distractor stimulus and a response is of theoretical interest. On the one hand, it increases our understanding of the nature of the inhibition process. Finding weaker distractor-response bindings in those conditions that are characterized by stronger inhibition processes would suggest that inhibition operates by preventing the processing of distractors or by deactivating distractor representations completely (Houghton \& Tipper, 1994). Conversely, finding that distractor-response bindings are independent of the strength of distractor inhibition would support the hypothesis that inhibition targets only specific facets of distractor representations (i.e., their associations with specific responses), thus preventing the access of activated 
distractors to response selection processes (Tipper \& Cranston, 1985; see also De Houwer et al., 2001; Frings \& Wentura, 2006; Fuentes et al., 1999; Tipper et al., 1994).

Furthermore, an investigation of the interactions between distractor inhibition and distractor-response binding would also be informative with regard to the nature of binding processes. Finding evidence for distractor-response bindings despite strong distractor inhibition would suggest that the binding of stimuli and responses occurs more or less in an automatic fashion and that it cannot be prevented by actively blocking the link between a distractor and its assigned response (Mayr et al., 2009). If, on the other hand, distractor-response bindings are eliminated or weakened in conditions that are characterized by strong distractor inhibition, this would suggest that binding requires attention (Treisman, 1999).

\section{Assessing distractor inhibition and distractor-response bindings: rationale of the present study}

In the present study, we investigated the processes of distractor inhibition and distractor-response binding for incompatible and neutral distractors within the same experiments. Manipulating the task relevance or interference potential of the distractors should have an influence on the strength of inhibition processes, and we wanted to know whether it also would affect the integration of distractors and responses into event files.

Traditionally, distractor inhibition effects are investigated with a sequential-priming paradigm: It is assumed that if an irrelevant stimulus is inhibited during target selection, inhibition persists from trial $n-1$ (the prime trial) to trial $n$ (the probe trial). Hence, if an irrelevant prime distractor is repeated as a target in the subsequent probe display (distractor-to-target repetition), responses are typically slowed down, because the internal stimulus representation still suffers from inhibition (Neill, 1977; Tipper, 1985). Similar negative effects of distractor-to-target repetitions have also been reported in the visual search literature (socalled "distractor previewing effects," "role reversal effects," or "switching effects"; Ariga \& Kawahara, 2004; Lamy, Antebi, Aviani, \& Carmel, 2008; Levinthal \& Lleras, 2008; Yashar \& Lamy, 2010).

Negative priming effects of distractor-to-target repetitions are the most commonly used measure of distractor inhibition. Nevertheless, several considerations speak against employing this measure of distractor inhibition in our study. First, distractor-to-target repetitions cannot be realized for neutral distractors, because by definition, neutral distractors are not assigned to a specific response, and thus cannot be presented as a target in the task. This makes it impossible to investigate inhibition effects for neutral distractors on the basis of distractor-to-target repetitions. Secondly, several alternative explanations that are unrelated to inhibition have been proposed to explain negative priming effects for distractor-to-target repetitions. For example, standard negative priming with distractor-to-target repetitions can also be explained with episodic retrieval processes (Neill, Valdes, Terry, \& Gorfein, 1992; Rothermund et al., 2005), feature mismatch (Park \& Kanwisher, 1994; MacDonald \& Joordens, 2000), or temporal discrimination processes (Milliken, Joordens, Merikle, \& Seiffert, 1998).

We thus used a different measure of distractor inhibition in our study that (a) allows for a comparison of the strengths of inhibition processes for incompatible and neutral distractors, and that (b) cannot easily be explained by alternative processes. An analysis of distractor-todistractor repetitions is well suited for this purpose. If a prime distractor is repeated as the probe distractor (distractor-to-distractor repetition), responses in the probe trial are generally faster, because selection of the probe target against an already inhibited distractor is easier (Frings \& Wühr, 2007; E. Neumann \& DeSchepper, 1991; Tipper \& Cranston, 1985; see also Lorch, Anderson, \& Well, 1984). Similar distractor repetition benefits have also been reported in the visual search literature and have been interpreted in terms of distractor inhibition (Lamy et al., 2008; Yashar \& Lamy, 2010).

Facilitative effects of distractor-to-distractor repetitions can be interpreted as a straightforward measure of distractor inhibition, because they cannot easily be explained in terms of perceptual priming, selection feature (mis)match, or temporal discrimination processes. Furthermore, distractor repetition benefits can be computed for neutral and incompatible distractors alike, because this measure does not require that distractors be presented as targets during the probe trial. This allows us to compare the strengths of inhibition effects for neutral and incompatible distractors via the same measure of distractor inhibition.

Another advantage of this measure is that distractorto-distractor repetition effects can be used not only to estimate processes of distractor inhibition, but also to compute a measure of distractor-response binding that is orthogonal to the distractor inhibition effect: Inhibition of a distractor is conceptualized as a selection process that is triggered by a response conflict between a target and a distractor. Its primary function is to prevent the distractor from influencing an ongoing response selection process, either by deactivating the distractor or by marking the distractor as response irrelevant. Inhibition is further assumed to carry over from the prime to the probe, facilitating responding if 
the prime distractor is reencountered as a distractor in the probe. Because inhibitory tagging ${ }^{1}$ or deactivation of distractors occurs independently of future response requirements, distractor inhibition is indicated by a main effect of distractor repetitions (distractor repetition benefit) that is independent of the response relation between prime and probe (Frings \& Wühr, 2007; E. Neumann \& DeSchepper, 1991). Distractor-response bindings, on the other hand, are indicated by an interaction of distractor repetition effects with response relation (Frings \& Rothermund, 2011; Frings et al., 2007; Giesen \& Rothermund, 2011; Rothermund et al., 2005). Specifically, repeating the distractor should facilitate responding in response repetition sequences (in which the distractor retrieves the correct response from the prime) but should delay responding if the prime and probe responses differ (because the distractor retrieves a response from the prime that is inappropriate in the probe). Stronger distractor repetition benefits for response repetition than for response change sequences thus indicate distractor-response binding and retrieval. Importantly, this interaction effect (computed as the difference between the distractor repetition benefits for response repetitions and response changes) is orthogonal to the main effect of distractor repetitions (computed as the average of the distractor repetition benefits across these two conditions) that is used to assess the strength of distractor inhibition. ${ }^{2}$ Analyzing distractor repetition effects thus allows us to investigate inhibition and distractor-response binding on the basis of the same type of repetition effect (distractor-to-distractor repetitions), while alternative explanations of the repetition effects can be ruled out (Rothermund et al., 2005).

The core focus of our study thus consisted of an analysis of the interactions of the distractor inhibition and distractorresponse binding effects with distractor type (Fig. 1 summarizes the core hypotheses and tests): (a) The two-way interaction of distractor repetition and distractor type allowed us to test whether the strengths of distractor inhibition effects differ for incompatible and neutral distractors. Distractor

\footnotetext{
${ }^{1}$ An episodic retrieval account that focuses on the retrieval of "donot-respond" tags (Neill et al., 1992) can also be classified as an inhibitory account, because it assumes that incompatible distractors are marked as irrelevant and are disconnected from response selection processes (see Milliken et al., 1998; Tipper, 2001), which closely corresponds to the view of how inhibition works that is advanced here. ${ }^{2}$ Computing the inhibition effect as the average of the distractor repetition effects across response repetition and response change sequences rests on the assumption that the facilitation and interference effects due to distractor-based retrieval of the prime response are of equal absolute magnitudes, and thus cancel out each other in the inhibition measure. Empirical support for this assumption has come from previous research showing that the facilitative effects of compatible and the interference effects of incompatible distractors are of approximately equal magnitudes in the flanker paradigm (Eriksen \& Eriksen, 1974).
}

Distractor inhibition

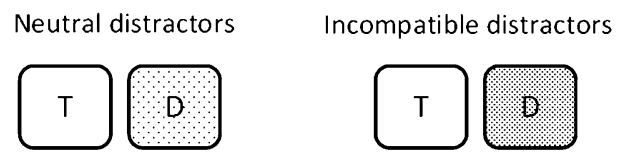

Hypothesis:

Stronger inhibition of incompatible distractors

Test:

Distractor repetition benefit $\mathrm{X}$ Distractor type

\section{Distractor-response binding}

Neutral distractors

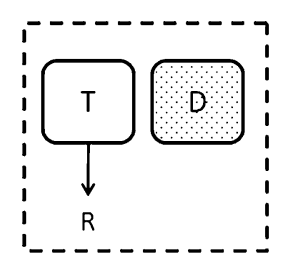

Hypotheses:

(a) No/weak distractor-response binding for incompatible distractors (b)Distractor-response binding for incompatible \& neutral distractors

Test:

Distractor repetition benefit $\mathrm{X}$ Response relation $\mathrm{X}$ Distractor type

Fig. 1 Illustration of core hypotheses and tests $(T=$ target stimulus, $\mathrm{D}=$ distractor, $\mathrm{R}=$ response). The shades of the grey areas indicate strengths of distractor inhibition (dark = strong inhibition). The boxes in dashed lines indicate binding of stimuli and responses in event files

repetition benefits were expected to be stronger for incompatible than for neutral distractors, indicating stronger inhibition effects for incompatible distractors. (b) The three-way interaction of distractor repetition, response relation, and distractor type allowed us to analyze whether distractor type has an influence on the strength of distractorresponse bindings. A significant three-way interaction would indicate that distractor-response bindings are modulated by distractor type (they may be eliminated or reduced for incompatible vs. neutral distractors). Finding equally strong effects of distractor-response binding for incompatible and neutral distractors (no three-way interaction) would indicate that inhibition and binding operate independently.

\section{Experiment 1}

In Experiment 1, participants' task was to identify a target stimulus by pressing one of four possible response keys and to ignore two simultaneously presented flanker stimuli for each prime and probe display. Distractor relation and response relation were manipulated independently and could either repeat or change across prime-probe sequences. Inherent to the identification task, response repetition 
implied that the identical target was presented as prime and probe, whereas response change implied that different targets were presented as prime and probe. Distractor type (incompatible vs. neutral distractors) was manipulated between subjects.

\section{Method}

Participants In total, 144 students of Friedrich Schiller University of Jena took part in Experiment 1. Two of these participants had to be excluded from all analyses because of excessive error rates $(>20 \%)$. Thus, the data of 142 (88 female, 54 male) participants were analyzed, 46 in the incompatible distractor condition, and 48 in each of the two neutral distractor conditions. Participants' mean age was 22.2 years, and they received $€ 2$ and a bar of chocolate for their participation. Experimental sessions lasted approximately $30 \mathrm{~min}$.

Materials Four different stimulus sets (JKLM, PQRT, 1234, and 5678) served as stimuli in the experiment. For each participant, one stimulus set was chosen as the target set (target sets were counterbalanced across participants), and each of the four stimuli of the target set was assigned to one of four different response keys on a response box that was connected to the computer via a parallel port. A paper tag was fixed immediately above each key on the response box, indicating which of the four target stimuli was assigned to which of the four response buttons for the particular participant. In the incompatible distractor condition, the distractor stimuli were drawn from the same set of stimuli as the targets; in the "neutral/different class" distractor condition, the distractor stimuli were drawn from opposing stimulus sets (numbers if the targets were letters, and vice versa). However, distractors in this condition were not only neutral with respect to the target identification task, but also belonged to a different stimulus category than the target stimuli. In order to control for this confound of stimulus class and distractor type, we employed a second neutral condition: In the "neutral/same class" distractor condition, the distractors were drawn from the other stimulus set within the same class of stimuli as the targets. For example, if JKLM constituted the target set, distractors were drawn from the PQRT set in order to guarantee that although distractors belonged to the same stimulus class as the targets (e.g., letters), the identification task could not be applied to them (i.e., the distractors were neutral with respect to the task). Targets and distractors were always presented in white font on a black computer screen. Each letter/digit was about $0.9 \mathrm{~cm}$ high and $0.4-0.8 \mathrm{~cm}$ wide. On each display, a stimulus triplet was presented (e.g., JKJ or 5M5), with the centrally presented stimulus as target and the first and third stimuli as flanking distractors. To reduce spatial selection of the target (Gibbons \& Frings, 2010), the stimulus triplets were presented randomly at one of four possible positions on the screen (left, right, above, or below the fixation cross). Hence, the positions of the stimulus triplets either repeated $(25 \%$ of all trials) or changed $(75 \%$ of all trials) from prime to probe; however, this factor did not interact with the factors of interest and was not incorporated into the analyses.

Design The experimental design consisted of the two within-subjects factors, distractor relation and response relation, and the between-subjects factor distractor type (see Table 1). Distractor relation was manipulated by either repeating the prime distractor in the probe (distractor repetition) or by presenting different distractor stimuli in the prime and probe trials of a sequence (baseline). Secondly, responses could either repeat or change between
Table 1 Sample stimuli of prime-probe sequences in Experiment 1
The sample stimuli are for targets belonging to Letter Set 1 $(\mathrm{J}, \mathrm{K}, \mathrm{L}, \mathrm{M}) . R R$ response repetition, $R C$ response change, $D R$ distractor repetition, $D C$ distractor change

\begin{tabular}{|c|c|c|c|c|}
\hline \multirow[b]{2}{*}{ Distractor Type } & \multicolumn{2}{|l|}{ Condition } & \multicolumn{2}{|c|}{ Sample sequences } \\
\hline & Response Relation & Distractor Relation & Prime & Probe \\
\hline \multirow[t]{4}{*}{ Incompatible distractor } & \multirow[t]{2}{*}{$\mathrm{RR}$} & DR & JKJ & $\mathrm{JKJ}$ \\
\hline & & DC & $\mathrm{JKJ}$ & MKM \\
\hline & \multirow[t]{2}{*}{$\mathrm{RC}$} & DR & JKJ & JLJ \\
\hline & & DC & $\mathrm{JKJ}$ & MLM \\
\hline \multirow[t]{4}{*}{ Neutral distractor/ same class } & \multirow[t]{2}{*}{$\mathrm{RR}$} & DR & PKP & PKP \\
\hline & & DC & PKP & QKQ \\
\hline & \multirow[t]{2}{*}{$\mathrm{RC}$} & DR & PKP & PLP \\
\hline & & DC & PKP & QLQ \\
\hline \multirow[t]{4}{*}{ Neutral distractor/ different class } & \multirow[t]{2}{*}{$\mathrm{RR}$} & DR & $1 \mathrm{~K} 1$ & $1 \mathrm{~K} 1$ \\
\hline & & DC & $1 \mathrm{~K} 1$ & $4 \mathrm{~K} 4$ \\
\hline & \multirow[t]{2}{*}{$\mathrm{RC}$} & DR & $1 \mathrm{~K} 1$ & $1 \mathrm{~L} 1$ \\
\hline & & DC & $1 \mathrm{~K} 1$ & $4 \mathrm{~L} 4$ \\
\hline
\end{tabular}


prime and probe (by repeating the target or not). Thirdly, distractor type was manipulated: Distractors were either incompatible (i.e., they were drawn from the same stimulus set as the targets), neutral/same class (i.e., they were drawn from the nontarget set within the same stimulus class as the targets), or neutral/different class (i.e., they were drawn from a nontarget set of the stimulus class opposite from that of the targets).

Procedure Participants were tested individually. Instructions were given on the screen. Participants were informed that in each trial, a stimulus triplet consisting of a target (central position) with two flanking distractors would be presented. For both prime and probe displays, participants' task was to identify the target stimulus by pressing the corresponding key on the response pad (keys were labeled according to the particular target set condition). Participants were instructed to keep their left and right middle fingers on the outer, and their left and right index fingers on the inner of the four response keys throughout the experiment. A fifth key, located in between both inner keys, was labeled "space" and served to start each prime-probe sequence via a thumbpress. Participants were reminded to respond as quickly and accurately as possible.

Each experimental prime-probe sequence was as follows (see Fig. 2): First, a fixation cross (+) appeared at the center of the screen until participants pressed the "space" key to start the prime-probe sequence. After $750 \mathrm{~ms}$, the prime display was presented, consisting of a stimulus triplet. The stimuli remained on the screen until participants pressed one of the four response keys. The screen was then cleared for $250 \mathrm{~ms}$. Subsequently, the probe display was presented, again consisting of a stimulus triplet, until participants pressed one of the four response keys. Then the screen was cleared again. After an intertrial interval of $900 \mathrm{~ms}$, the fixation cross for the next trial sequence appeared.

Participants first performed a practice block of 25 prime-probe sequences in which they received feedback regarding the correctness of their performance after each sequence. The experiment started if $60 \%$ or more of the responses in the practice trials were correct; otherwise, the practice block was repeated.

Participants then performed 256 experimental primeprobe sequences that were constructed with respect to the experimental factors (see Table 1) and with certain restrictions: (a) Distractors in the incompatible distractor condition were always different from the target. (b) Prime targets were never repeated as a distractor in the probe display, and prime distractors were never repeated as a target in the probe display in the incompatible distractor condition. (c) Response repetition (i.e., repetition of the target; RR) occurred on $25 \%$ of all trials, whereas response changes (RC) occurred on $75 \%$ of all trials. (d) Distractor repetitions (DR) occurred on $25 \%$ of all trials, leaving $75 \%$ of all trials with distractor change (DC).

Halfway through the experiment, participants were given a short break. At the end of the experiment, they were asked whether they had used any strategies during the task. Finally, participants were thanked, debriefed, and rewarded.

\section{Results}

Our first aim was to test whether distractor repetition benefits are stronger for incompatible than for neutral distractors. Therefore, we predicted that an interaction of distractor repetition and distractor type would be significant for the contrast between the incompatible and the two
Fig. 2 Schematic display of the trial structure in Experiments 1 and 2. For each prime-probe sequence, participants reacted via keypress to the centrally presented target stimulus by performing an identification task (Exp.1) or a categorization task (Exp.2). The stimuli are not drawn to scale

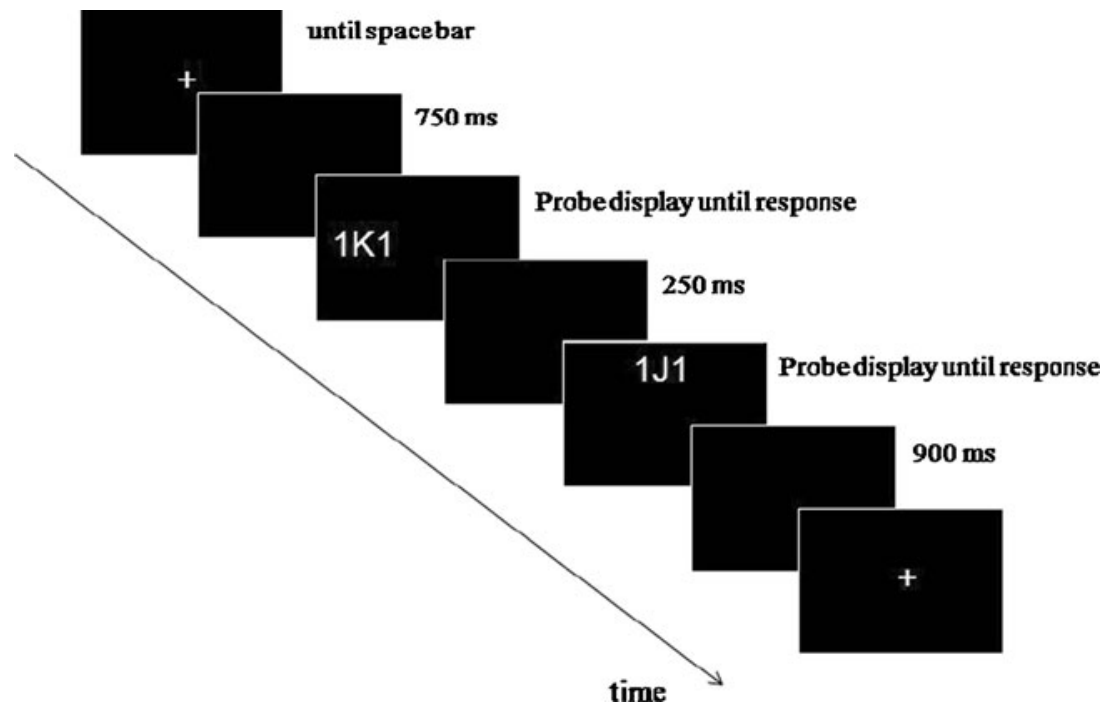


neutral distractor conditions. We did not predict any difference in distractor repetition benefits between the two neutral conditions ("neutral/same class" vs. "neutral/different class").

Our second aim was to test whether incompatible distractors were less likely than neutral distractors to be integrated into an event file, which should be reflected in a significant three-way interaction of distractor relation, response relation, and distractor type. Alternatively, the absence of a three-way interaction would indicate that distractor-response binding effects were unaffected by the distractor type manipulation. Again, the crucial test of this hypothesis referred to the contrast between the incompatible and the two neutral distractor conditions. No effects were predicted regarding the contrast between the two neutral distractor conditions.

Probe reaction times (RTs) were the primary dependent variable. Only prime-probe sequences with correct responses in the prime and probe trials were considered, and thus $5.2 \%$ of all trials were excluded from the RT analyses. Furthermore, limits for outlier values were computed individually for each participant: RTs to the probe display that were below $250 \mathrm{~ms}$ or more than 1.5 interquartile ranges above the third quartile of the individual distribution of probe RTs (3.2\%; Tukey, 1977) were discarded from the analyses.

Average probe RTs were computed for every condition of the factorial design and separately for each participant (see Table 2 for the means and standard deviations). These means were then entered into a 2 (distractor relation: repetition vs. change) $\times 2$ (response relation: repetition vs. change) $\times 3$ (distractor type: incompatible vs. neutral/same class vs. neutral/different class) mixed models ANOVA.

The main effect of distractor repetition was significant, $F$ $(1,139)=242.15, p<.001, \eta_{\mathrm{p}}{ }^{2}=.64$, indicating an overall distractor repetition benefit. Participants responded faster for prime-probe sequences with distractor repetitions (595 ms) than with distractor changes $(618 \mathrm{~ms})$. The other two main effects were also significant: Participants responded faster in response repetition trials $(527 \mathrm{~ms})$ as compared to response change trials $(686 \mathrm{~ms}), F(1,139)=$ 479. $43, p<.001, \eta_{\mathrm{p}}{ }^{2}=.78$, and responses were slower in the incompatible distractor condition $(650 \mathrm{~ms})$ than in the two neutral distractor conditions (586 and $583 \mathrm{~ms}$ for neutral distractors from the same and from the different class, respectively), $F(2,139)=9.74, p<.001, \eta_{\mathrm{p}}{ }^{2}=.12$, indicating stronger interference for incompatible than for neutral distractors.

The main effects were further qualified by several interactions: First, the predicted interaction of distractor repetition and distractor type emerged, $F(2,139)=14.37$, $p<.001, \eta_{\mathrm{p}}{ }^{2}=.17$, indicating that the strength of distractor inhibition differed for incompatible and neutral distractors. Conforming with our prediction, planned contrasts revealed that the distractor repetition benefit was stronger for incompatible distractors $(\Delta=33 \mathrm{~ms}, S D=17 \mathrm{~ms})$ than for either the neutral/same class distractor condition $(\Delta=$ $19 \mathrm{~ms}, S D=16 \mathrm{~ms})$ or the neutral/different class distractor condition $(\Delta=15 \mathrm{~ms}, S D=18 \mathrm{~ms}), t(139)=5.19, p<.001$. Distractor repetition benefits in the two neutral distractor conditions did not differ significantly from each other, $t$ $(139)=1.35, p>.17$.

Table 2 Means and standard deviations of reaction times (RTs, in milliseconds) and error rates for the probe display, Experiment 1

\begin{tabular}{|c|c|c|c|c|c|c|}
\hline \multirow[b]{2}{*}{ Distractor type } & \multicolumn{2}{|l|}{ Condition } & \multicolumn{2}{|l|}{ RT } & \multicolumn{2}{|c|}{$\%$ Error } \\
\hline & Response relation & Distractor relation & M & $\mathrm{SD}$ & $\mathrm{M}$ & SD \\
\hline \multirow[t]{4}{*}{ Incompatible distractor $(n=46)$} & \multirow[t]{2}{*}{$\mathrm{RR}$} & DR & 525 & 70 & 0.8 & 2.2 \\
\hline & & $\mathrm{DC}$ & 586 & 75 & 1.1 & 2.3 \\
\hline & \multirow[t]{2}{*}{$\mathrm{RC}$} & DR & 741 & 130 & 3.9 & 3.9 \\
\hline & & $\mathrm{DC}$ & 748 & 123 & 3.6 & 2.8 \\
\hline \multirow[t]{4}{*}{ Neutral distractor/ same class $(n=48)$} & \multirow[t]{2}{*}{$\mathrm{RR}$} & DR & 485 & 57 & 0.1 & 0.9 \\
\hline & & $\mathrm{DC}$ & 533 & 59 & 0.7 & 1.5 \\
\hline & \multirow[t]{2}{*}{$\mathrm{RC}$} & DR & 669 & 110 & 4.0 & 3.9 \\
\hline & & $\mathrm{DC}$ & 660 & 106 & 2.6 & 2.4 \\
\hline \multirow[t]{4}{*}{ Neutral distractor/ different class $(n=48)$} & \multirow[t]{2}{*}{$\mathrm{RR}$} & DR & 498 & 56 & 0.7 & 3.0 \\
\hline & & $\mathrm{DC}$ & 536 & 58 & 1.0 & 1.7 \\
\hline & \multirow[t]{2}{*}{$\mathrm{RC}$} & $\mathrm{DR}$ & 653 & 119 & 3.4 & 2.8 \\
\hline & & DC & 645 & 114 & 3.4 & 2.7 \\
\hline
\end{tabular}

$R R$ response repetition, $R C$ response change, $D R$ distractor repetition, $D C$ distractor change 
We also found an interaction of distractor relation and response relation, $F(1,139)=248.70, p<.001, \eta_{\mathrm{p}}{ }^{2}=.64$, replicating the general phenomenon of distractor-response binding and retrieval. That is, repetition of the prime distractor in the probe display led to RT benefits of $M=$ $49 \mathrm{~ms}(S D=32 \mathrm{~ms})$ for response repetition sequences, which differed significantly from zero, $t(141)=18.16, p<.001$, but responding was delayed by $M=-4 \mathrm{~ms}(S D=22 \mathrm{~ms})$ for response alternation sequences, $t(141)=-1.97, p=.05$. Importantly, there was no indication of a three-way interaction between distractor relation, response relation, and distractor type, $F<1$. This finding indicates that the distractor-response binding effects were of equal magnitudes for incompatible and neutral distractors (see Fig. 3). Post hoc comparisons revealed that the three-way interaction was not significant for the contrast between incompatible and neutral distractors $(t<1)$, nor for the contrast between the two neutral distractor conditions $[t(139)=-1.36, p>.17]$. The interaction of response relation and distractor type was significant as well, $F(2,139)=5.13, p<.01, \eta_{\mathrm{p}}{ }^{2}=.07$, but was not of theoretical interest and is not discussed further.

The same mixed models ANOVA on mean error rates (see Table 2) revealed that on average, participants made fewer errors in sequences with response repetition $(0.7 \%)$ than with response alternations $(3.5 \%), F(1,139)=160.65$, $p<.001, \eta_{\mathrm{p}}{ }^{2}=.53$. This main effect of response relation was further specified by an interaction with distractor relation, $F(1,139)=9.31, p<.001, \eta_{\mathrm{p}}^{2}=.06$, showing that repetition of the prime distractor in the probe display led to an error reduction of $M=-0.4 \%(S D=2.2 \%)$ in response repetition sequences, which was significantly different from zero, $t(141)=-2.11, p<.05$, but increased

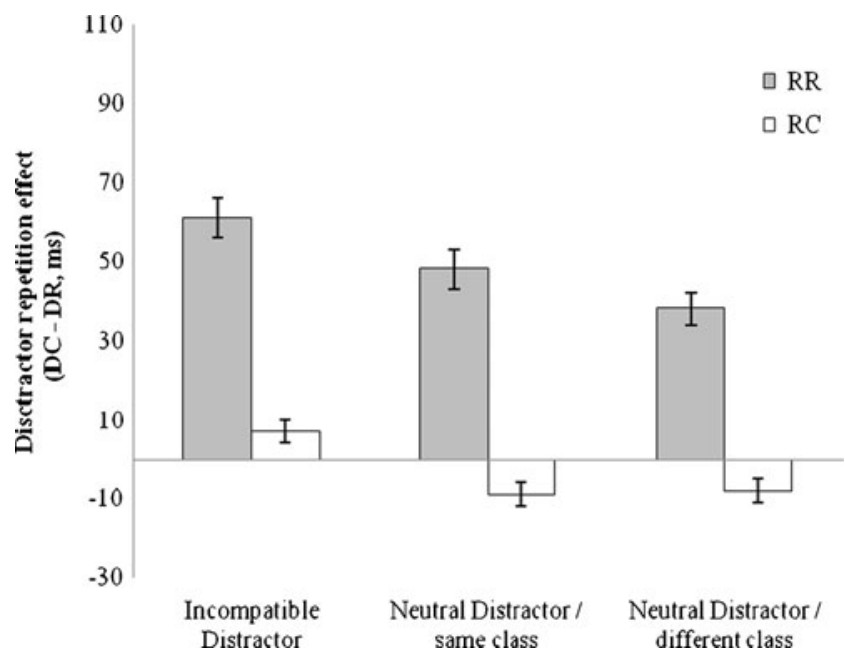

Fig. 3 Distractor repetition effects (distractor change [DC] minus distractor repetition [DR], in milliseconds) as a function of response relation (response repetition [RR] vs. response change [RC]) and distractor type in Experiment 1. Error bars depict the standard errors of the means error rates by $M=0.6 \%(S D=3.2 \%)$ for sequences with response alternations, $t(141)=2.21, p<.05$. All other effects failed to reach conventional levels of significance (all $F \mathrm{~s}<2.4, p \mathrm{~s}>.10$ ).

\section{Discussion}

In Experiment 1, we investigated the modulating influence of distractor type on distractor inhibition and its consequences for the integration of distractors and responses into event files. As predicted, distractor inhibition was stronger for incompatible than for neutral distractors and did not differ between the two neutral distractor conditions. Distractor repetition benefits were stronger for incompatible than for neutral distractors, indicating that it was easier to process the probe display if the distractor still suffered from inhibition that had been initiated during the prime (Frings \& Wühr, 2007; Lamy et al., 2008; Yashar \& Lamy, 2010).

Additionally, we found evidence for automatic storage and retrieval of distractor-response bindings in our study. Repeating the prime distractor as a distractor in the probe had a positive effect on performance for response repetition sequences, but this turned into a cost if the responses changed from prime to probe. Thus, our findings replicate previous evidence showing that the prime distractor is integrated in the prime episode and automatically retrieves the prime response if it is encountered again in a subsequent trial (Frings \& Rothermund, 2011; Frings et al., 2007; Giesen \& Rothermund, 2011; Rothermund et al., 2005). Of central interest for our study was the finding that the strengths of the distractor-response binding effect did not differ between incompatible and neutral distractors, indicating that the distractor was integrated into the $\mathrm{S}-\mathrm{R}$ episode of the prime trial, regardless of the strength of distractor inhibition. This finding supports the assumption that distractor inhibition and distractor-response binding operate independently of each other.

Experiment 1 had one caveat, though. In the identification task that was used in this experiment, the factor response relation was confounded with target relation: Response repetitions implied that the same target stimulus was presented in the prime and probe displays, whereas response alternations implied that the target changed as well. The distractor repetition benefits that obtained within the target repetition trials might thus have reflected processes that were specific for complete stimulus repetitions (prime target and prime distractor repeated in the probe display). For example, complete stimulus repetitions might tend to shortcut the response selection stage in the probe, because participants tend to rely on a "bypass rule" that suggests a repetition of the previous response in the case of a complete stimulus repetition (Fletcher \& Rabbitt, 1978; see also Hommel \& Colzato, 2004; Pashler \& Baylis, 
1991). In order to rule out alternative explanations of our findings in terms of processes that are elicited by complete stimulus repetitions, we conducted another experiment with a categorization task that allowed us to manipulate the response relation independently of target repetitions (Frings et al., 2007). In this task, two stimuli were always assigned to the same response key, so that responses could be repeated although the target changed from prime to probe. By restricting the analysis to only those trials on which a different target stimulus was presented on both the prime and probe trials, any influence of identical stimulusresponse repetitions on the results would be eliminated.

\section{Experiment 2}

\section{Method}

Participants A group of 84 students at Friedrich Schiller University of Jena took part in the experiment. One of the participants made $61 \%$ errors and had to be excluded from all analyses. Thus, the data of 83 (49 female, 34 male) participants were analyzed. Participants' mean age was 23.4 years $(S D=4.4)$. The experiment duration and payment of participants paralleled those aspects of Experiment 1.

Materials, design, and procedure The materials, design, and procedure were identical to those aspects of Experiment 1, except for the following alterations: The task was changed to a categorization task, now with eight stimuli mapped to the four keys of the response pad. Therefore, we merged Letter Sets 1 and 2 and Digit Sets 1 and 2 to one letter and one digit set, which now consisted of eight stimuli each. For each set, two stimuli were assigned to the same response key. Two different stimulus-response key assignments, counterbalanced across participants, were realized for each set of stimuli (JK-LM-PQ-RT vs. LRTQ-MK-PJ, and $12-34-56-78$ vs. 38-25-74-61). ${ }^{3}$ Target set (letters vs. digits) was counterbalanced across participants. Because both neutral distractor conditions yielded identical results in Experiment 1, we dropped the neutral/ same class distractor condition. For half of the participants, distractors were drawn from the same set as the targets (incompatible distractor condition; i.e., the distractors were letters/digits if the targets were letters/digits), whereas for the remaining participants, the distractors were drawn from the other set (neutral distractor condition, corresponding to the former neutral/different class distractor condition; i.e., the distractors were digits/letters if the targets were letters/

\footnotetext{
${ }^{3}$ Stimulus assignments to response keys did not interact with the factors of interest, and hence all analyses were performed with this factor collapsed.
}

digits). The former response repetition condition now consisted either of trials on which the prime target was either identically repeated in the probe display (T-ID; e.g., $1 \mathrm{~K} 1-1 \mathrm{~K} 1$; see Table 3 for examples of the complete factorial design) or of trials on which the prime and probe targets were different but shared the same category response key (T-SC; e.g., 1M1-1K1). The former response change condition now corresponded to trials on which the prime and probe targets had different category response keys (T-DC; e.g., 1P1-1K1). A similar logic applies to the distractor relation factor: We presented the identical distractor in prime and probe displays (D-ID; e.g., 1K1$1 \mathrm{~K} 1)$ or presented two different distractors, which either shared the same category response key (D-SC; e.g., 6K6$1 \mathrm{~K} 1$ ) or had different category response keys (D-DC; e.g., $7 \mathrm{~K} 7-1 \mathrm{~K} 1)$. Because we were interested in the binding and retrieval of distractor and response information, we contrasted the condition with a repetition of the same distractor (D-ID) with a baseline, in which a different distractor was presented in the probe that shared the same category response key with the prime distractor (D-SC). This comparison focused entirely on the stimulus repetition of the distractor and was not confounded with differences in the responses to which distractors are assigned.

Participants performed 240 experimental prime-probe sequences that were constructed with respect to the experimental factors (see Table 3) and had the same constraints as in Experiment 1. Within the response repetition trials, $50 \%$ were trials with repetition of identical targets (T-ID), and 50\% were trials with different targets from the same response category (T-SC). By definition, all response change trials were trials with targets from different response categories (T-DC). For each level of the target relation factor, the three distractor relation levels occurred equally often; that is, one third were trials with identical distractors (D-ID), with distractors from the same response category (D-SC), and with distractors from different response categories (D-DC), respectively.

\section{Results}

As in Experiment 1, we expected distractor repetition benefits to be stronger for incompatible than for neutral distractors, as indicated by a significant distractor relation $\times$ distractor type interaction. Secondly, the design modifications in Experiment 2 allowed us to analyze distractor-response binding and retrieval with a more stringent test - that is, independently of identical stimulus repetitions: Trials with identical target repetitions did not enter into the analyses, resulting in a design without complete stimulus repetitions. We expected to replicate the interaction of distractor relation and response relation in this experiment, using only the T-SC and T-DC conditions as 
Table 3 Sample stimuli of prime-probe sequences in Experiment 2
The sample stimulus triplets are for the unsystematic assignment of stimuli to response keys (i.e., LR-TQ-MK-PJ and 38-25-

74-61). RR, response repetition; $\mathrm{RC}$, response change. T-ID, identical target repetition; T-SC, target with the same category response key; T-DC, target with different category response key. D-ID, identical distractor repetition; D-SC, distractor with same category response key; D-DC, distractor with different category response key

\begin{tabular}{|c|c|c|c|c|c|}
\hline \multirow{3}{*}{$\frac{\text { Distractor type }}{\text { Incompatible distractor }}$} & \multicolumn{3}{|c|}{ Condition } & \multicolumn{2}{|c|}{ Sample sequences } \\
\hline & \multicolumn{2}{|c|}{ Response relation } & \multirow{2}{*}{$\begin{array}{l}\text { Distractor relation } \\
\text { D-ID }\end{array}$} & \multirow{2}{*}{$\begin{array}{l}\text { Prime } \\
\text { PKP }\end{array}$} & \multirow{2}{*}{$\begin{array}{l}\text { Probe } \\
\text { PKP }\end{array}$} \\
\hline & $\mathrm{RR}$ & T-ID & & & \\
\hline & & & D-SC & $\mathrm{JKJ}$ & PKP \\
\hline & & & D-DC & TKT & PKP \\
\hline & $\mathrm{RR}$ & T-SC & D-ID & PMP & PKP \\
\hline & & & D-SC & JMJ & PKP \\
\hline & & & D-DC & TMT & PKP \\
\hline & $\mathrm{RC}$ & T-DC & D-ID & PLP & PKP \\
\hline & & & D-SC & JLJ & PKP \\
\hline & & & D-DC & TLT & PKP \\
\hline \multirow[t]{9}{*}{ Neutral distractor } & $\mathrm{RR}$ & T-ID & D-ID & $1 \mathrm{~K} 1$ & $1 \mathrm{~K} 1$ \\
\hline & & & D-SC & $6 \mathrm{~K} 6$ & $1 \mathrm{~K} 1$ \\
\hline & & & D-DC & $2 \mathrm{~K} 2$ & $1 \mathrm{~K} 1$ \\
\hline & $\mathrm{RR}$ & T-SC & D-ID & $1 \mathrm{M} 1$ & $1 \mathrm{~K} 1$ \\
\hline & & & D-SC & $6 \mathrm{M} 6$ & $1 \mathrm{~K} 1$ \\
\hline & & & D-DC & $2 \mathrm{M} 2$ & $1 \mathrm{~K} 1$ \\
\hline & $\mathrm{RC}$ & T-DC & D-ID & $1 \mathrm{~L} 1$ & $1 \mathrm{~K} 1$ \\
\hline & & & D-SC & $6 \mathrm{~L} 6$ & $1 \mathrm{~K} 1$ \\
\hline & & & D-DC & $2 \mathrm{~L} 2$ & $1 \mathrm{~K} 1$ \\
\hline
\end{tabular}

operationalizations of response repetition and response change, respectively. Given the finding of distractor-response bindings in general, we were also interested in whether the finding of equally strong bindings for incompatible and neutral distractors with prime responses would also replicate.

According to the same criteria as in Experiment 1, 9.1\% of all trials were discarded from analyses. Average probe RTs were computed for every condition of the factorial design and separately for each participant (see Table 4). These means were then entered into a 2 (response relation: same response [nonidentical target from same response category, T-SC] vs. different response [target from different response category, T$\mathrm{DC}]) \times 2$ (distractor repetition: identical distractor [D-ID] vs. distractor from same response category [D-SC]) $\times 2$ (distractor type: incompatible vs. neutral) mixed models ANOVA. ${ }^{4}$ The main effect of distractor repetition was significant, $F(1,81)=9.23, p<.01, \eta_{\mathrm{p}}{ }^{2}=.10$ : Probe responses were faster for prime-probe sequences with identical distractors $(851 \mathrm{~ms})$ than for sequences with distractors from the same response category $(872 \mathrm{~ms})$. The main effect of distractor type was also significant, $F(1,81)=$ $5.24, p<.05, \eta_{\mathrm{p}}{ }^{2}=.06$. Responses were generally slower in the incompatible distractor condition $(913 \mathrm{~ms})$ than in the

\footnotetext{
${ }^{4}$ The aim of Experiment 2 was to rule out any influence of full stimulus repetitions on the results. To obtain a maximally focused test that did not contain identical combinations of target and distractor in the prime and probe displays, the T-ID condition was thus excluded from this analysis. The results of the full analysis, including the T-ID condition, however, revealed the same pattern of effects.
}

neutral distractor condition $(811 \mathrm{~ms})$. The main effect of response relation was not significant, $F<1$.

These main effects were further qualified by several interactions: The predicted interaction of distractor repetition and distractor type emerged, $F(1,81)=4.31, p<.05, \eta_{\mathrm{p}}{ }^{2}=$ .05. A significant distractor repetition benefit was found for incompatible distractors $(M=-35 \mathrm{~ms}), t(81)=3.60, p<$ .001 , whereas for neutral distractors, no distractor repetition benefit obtained $(M=-7 \mathrm{~ms}), t<1$, indicating stronger inhibition effects for incompatible than for neutral distractors. The interaction of distractor repetition and target relation was also significant, $F(1,81)=9.21, p<.01$, $\eta_{\mathrm{p}}{ }^{2}=.10$. Distractor repetition had a facilitative effect on responding in response repetition sequences $(M=-40 \mathrm{~ms}), t$ $(82)=-3.11, p<.01$, but not if the response changed from prime to probe $(M=-1 \mathrm{~ms}), t<1$, indicating distractorbased retrieval of the prime response. As in the previous experiment, the three-way interaction of distractor relation, target relation, and distractor type was not significant, $F(1$, $81)=1.59, p>.21$, indicating that the effects of distractorresponse binding and retrieval did not differ for responseincompatible and neutral distractors ${ }^{5}$ (see Fig. 4). In addition,

\footnotetext{
${ }^{5}$ The numerical pattern of distractor repetition effects in Experiment 2 suggests that, if anything, the effects of distractor-based retrieval of the prime response were even somewhat stronger for incompatible distractors. Given the nonsignificant three-way interactions in both Experiments 1 and 2, we do not want to put too much weight on this finding, but clearly, the findings do not support the view that stronger inhibition leads to a reduction of distractor-response binding.
} 
Table 4 Sample means and standard deviations of reaction times (RTs, in milliseconds) and error rates in the probe display, Experiment 2
$R R$ response repetition, $R C$ response change, $T-I D$ identical target repetition, $T-S C$ target with the same category response key, $T-D C$ target with different category response key, $D-I D$ identical distractor repetition, $D-S C$ distractor with same category response key, $D-D C$ distractor with different category response key

\begin{tabular}{|c|c|c|c|c|c|c|c|}
\hline \multirow[b]{2}{*}{ Distractor Type } & \multicolumn{3}{|l|}{ Condition } & \multicolumn{2}{|l|}{ RT } & \multicolumn{2}{|c|}{$\%$ Error } \\
\hline & Response & Relation & Distractor Relation & M & $\mathrm{SD}$ & $\mathrm{M}$ & SD \\
\hline \multirow[t]{9}{*}{ Incompatible distractor $(n=41)$} & $\mathrm{RR}$ & T-ID & D-ID & 546 & 77 & 0.7 & 2.6 \\
\hline & & & $\mathrm{D}-\mathrm{SC}$ & 603 & 100 & 0.5 & 2.2 \\
\hline & & & D-DC & 599 & 86 & 0.5 & 2.1 \\
\hline & $\mathrm{RR}$ & T-SC & D-ID & 897 & 260 & 4.6 & 7.4 \\
\hline & & & $\mathrm{D}-\mathrm{SC}$ & 959 & 286 & 7.1 & 10.1 \\
\hline & & & $\mathrm{D}-\mathrm{DC}$ & 917 & 261 & 6.8 & 11.1 \\
\hline & $\mathrm{RC}$ & T-DC & D-ID & 894 & 175 & 4.6 & 6.0 \\
\hline & & & D-SC & 902 & 176 & 3.1 & 4.8 \\
\hline & & & D-DC & 898 & 171 & 2.6 & 3.1 \\
\hline \multirow[t]{9}{*}{ Neutral distractor $(n=42)$} & $\mathrm{RR}$ & T-ID & D-ID & 555 & 107 & 0.0 & 0.0 \\
\hline & & & $\mathrm{D}-\mathrm{SC}$ & 578 & 93 & 0.7 & 2.6 \\
\hline & & & D-DC & 576 & 98 & 0.0 & 0.0 \\
\hline & $\mathrm{RR}$ & T-SC & D-ID & 778 & 212 & 6.4 & 7.9 \\
\hline & & & $\mathrm{D}-\mathrm{SC}$ & 796 & 231 & 5.5 & 8.0 \\
\hline & & & D-DC & 797 & 219 & 8.3 & 9.1 \\
\hline & $\mathrm{RC}$ & T-DC & D-ID & 836 & 182 & 3.9 & 3.7 \\
\hline & & & D-SC & 832 & 183 & 3.1 & 3.3 \\
\hline & & & $\mathrm{D}-\mathrm{DC}$ & 828 & 181 & 2.9 & 3.6 \\
\hline
\end{tabular}

we observed a significant interaction of target relation and distractor type, $F(1,81)=9.02, p<.01, \eta_{\mathrm{p}}{ }^{2}=.10$, that was not of theoretical interest, and thus is not discussed further.

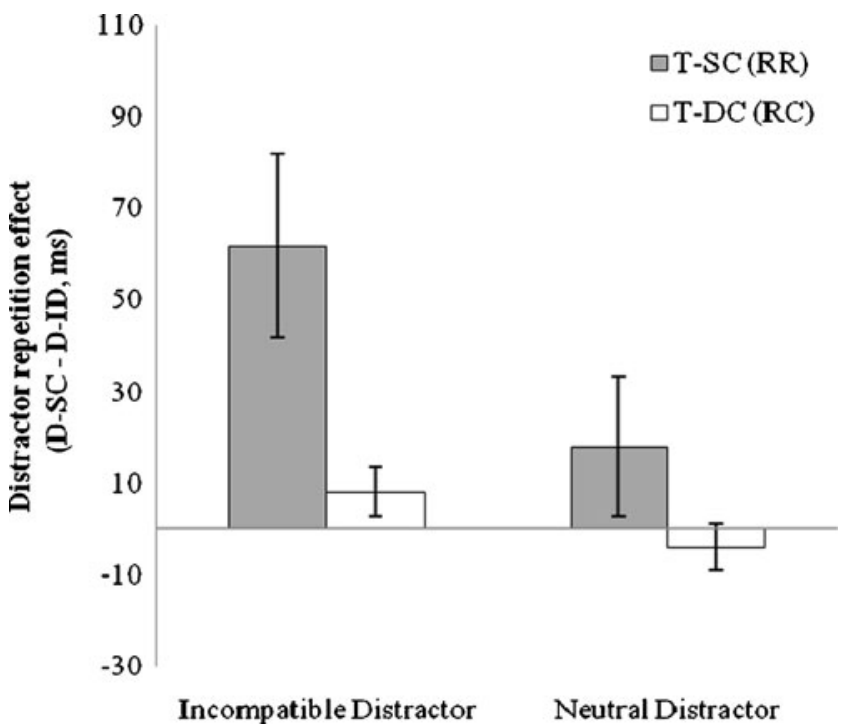

Fig. 4 Distractor repetition effects (distractor change [D-SC; different distractor of the same response category] minus distractor repetition [D-ID; identical distractor], in milliseconds) as a function of response relation (response repetition [RR/T-SC: different target of the same response category] vs. response change [RC/ T-DC: target of a different response category]) and distractor type in Experiment 2. Error bars depict the standard errors of the means
The same mixed models ANOVA on error rates (see Table 4) revealed a main effect of response relation, $F(1$, $81)=11.94, p<.01, \eta_{\mathrm{p}}{ }^{2}=.13$, indicating that somewhat more errors were made in the case of response repetitions (T-SC, 5.90\%) than in the case of response changes (T-DC, $3.67 \%$ ). All other effects were nonsignificant (all $F_{\mathrm{S}}<2.75$, ps $>.10)$.

\section{Discussion}

In Experiment 2, we used a categorization task instead of an identification task, which allowed us to manipulate the response relation independently of target repetitions (i.e., different target stimuli were presented for response repetition and response change sequences; Frings et al., 2007). The results of Experiment 2 replicated the pattern of findings from Experiment 1: Again, response-incompatible distractors were subject to stronger inhibition than were neutral distractors, indicated by the significant distractor repetition benefits (Frings \& Wühr, 2007; Lamy et al., 2008; Yashar \& Lamy, 2010) that obtained for incompatible but not for neutral distractors. Regarding distractor-response bindings, however, we found no evidence for differences between incompatible and neutral distractors. Distractors were integrated with responses, as indicated by an interaction of distractor relation and response relation (distractor repetitions facilitated responding in the case of response repetitions, but not in the case of response changes; Frings \& Rothermund, 
2011; Frings et al., 2007; Giesen \& Rothermund, 2011; Rothermund et al., 2005). This effect emerged for both incompatible and neutral distractors alike, supporting the hypothesis that distractors become associated with simultaneously executed responses, irrespective of whether the distractors are inhibited. Although distractors from the relevant target set have more potential to interfere with response selection, and consequently become inhibited, this did not affect distractor-response binding processes: Both types of distractors were equally likely to be integrated in the resulting event file.

\section{General discussion}

Two experiments were conducted in which we investigated the interplay between processes of selective attention and distractor-response bindings. We employed a distractor-todistractor repetition paradigm (Frings \& Rothermund, 2011; Frings et al., 2007; Giesen \& Rothermund, 2011; Rothermund et al., 2005) and manipulated whether the task could be applied to targets only (i.e., distractors were not related to specific responses, the neutral distractor condition) or to both targets and distractors (i.e., distractors were drawn from the same set as the target stimuli, the incompatible distractor condition). In both experiments, we found consistent evidence that this manipulation was successful in modulating processes of selective attention: Distractor repetition benefits, which reflect persisting inhibition of the prime distractor (Frings \& Wühr, 2007; Lamy et al., 2008; E. Neumann \& DeSchepper, 1991; Tipper \& Cranston, 1985; Yashar \& Lamy, 2010), were always stronger for incompatible than for neutral distractors. ${ }^{6}$ These findings support the notion that inhibitory processes respond "reactively" to the

\footnotetext{
${ }^{6}$ Distractor repetition benefits might also reflect processes other than inhibition. In particular, it can be argued that repeated distractors can be identified and rejected more easily as distractors because they are already bound to the feature that defines distractors (in this study, location). This explanation is reminiscent of the feature mismatch theory (MacDonald \& Joordens, 2000; Park \& Kanwisher, 1994), which postulates that binding a stimulus to the distractor feature delays processing of that stimulus if it is reencountered with another feature (i.e., as a target). In an analogous fashion, feature matches might facilitate the processing of repeated distractors because an already-established stimulus-feature binding can be retrieved from memory. However, findings from the negative-priming literature have demonstrated that feature (mis)match does not play an important role in the explanation of priming effects (e.g., Mackintosh, Mathews, \& Holden, 2002; Mayr, Hauke, Buchner \& Niedeggen, 2008; Tipper \& Cranston, 1985). In addition, the feature (mis)match account does not offer a straightforward interpretation of why the distractor repetition benefits should differ between incompatible and neutral distractors. Thus, although feature matches are a logical alternative to explain distractor repetition benefits, this seems not to be a likely explanation of the distractor repetition benefits in our study.
}

demands of action control by being sensitive to the interference potential of the distractors (De Houwer et al., 2001; Frings \& Wentura, 2006; Grison \& Strayer, 2001; Houghton et al., 1996; Levinthal \& Lleras, 2008; Tipper et al., 1994). Our study is the first to provide evidence for stronger inhibition of incompatible distractors on the basis of differences in distractor repetition benefits. Using distractorto-distractor repetition effects as a measure of inhibition has the advantage that alternative explanations of the effect are less plausible than for standard negative-priming effects that are based on distractor-to-target repetitions (see above).

The central interest of our study concerned the question of whether differences in inhibition regarding incompatible and neutral distractors affected the integration of the distractors and responses into event files. The results that were obtained in the two experiments yielded consistent evidence that this is not the case. Although responseincompatible distractors were subject to stronger inhibition, this did not prevent their integration with response information into event files. Instead, effects of distractorresponse bindings occurred with equal strengths for both incompatible and neutral distractors.

The conclusion that differences in inhibition did not affect distractor-response bindings rests on the interpretation of the nonsignificant three-way interaction of distractor relation, response relation, and distractor type. To substantiate this interpretation, we conducted post hoc power analyses to estimate the chances that we would have detected a difference in the strength of binding effects if it existed (power analyses were conducted with G*Power 3; Faul, Erdfelder, Lang, \& Buchner, 2007). The probability to detect a medium-sized effect $(f=.25)$ was $1-\beta>.99$ for Exp. $1(N=142)$, and $1-\beta>.99$ for Exp. $2(N=83)$. Even a small effect $(f=.10)$ would have been detected with sufficient power (Exp. 1: $1-\beta=.93$; Exp. $2: 1-\beta=.81$ ). With respect to our experiments, it thus seems safe to say that we did not overlook any differences in the strength of binding processes between neutral and incompatible distractors that were of at least medium size.

Although previous studies have reported evidence for distractor-response bindings of response-incompatible distractors (Frings \& Rothermund, 2011; Frings et al., 2007; Giesen \& Rothermund, 2011; Hommel, 2005; Mayr \& Buchner, 2006; Mayr et al., 2009), our study is the first that varied the strength of distractor inhibition within the same experiment, using the same type of distractor repetition effects to assess inhibition and binding processes. This allowed for a systematic investigation of the relation between the strength of inhibition and distractor integration into event files within a single paradigm. No such relation emerged in our experiments: Despite the fact that we obtained significant differences in the strengths of inhibition for response-incompatible and neutral distractors, the 
strengths of distractor-response binding effects did not differ between these two conditions. Our findings thus provide evidence that selective inhibition and binding processes are independent mechanisms. ${ }^{7}$

This view is consistent with Tipper et al.'s (1994) proposal of "flexible inhibition" that does not prevent processing of distractor information. Accordingly, inhibition processes prevent a translation of distracting stimuli into their associated responses, but do not prevent processing of the distractors per se (Tipper \& Cranston, 1985). A study by Fuentes et al. (1999) is particularly interesting, because it revealed additional information regarding the mechanism of inhibition. Using an inhibition-of-return paradigm, Fuentes et al. demonstrated that inhibition temporarily "disconnects" the distractor that is presented at an inhibited location from accessing its associated response, but apparently does not prevent processing of the distractor (distractor interference and priming effects were not neutralized, but were completely reversed in the inhibited location). Hence, the distractor appears to have been associated with an inhibitory tag that prevented its triggering of associated responses but did not prevent it from being integrated with responses into an event file.

\section{Theoretical implications}

Our findings contain important consequences for the understanding of binding processes that seem to differ from what some other researchers assumed. These previous studies predominantly conceptualized binding and inhibito-

\footnotetext{
$\overline{7}$ The incompatible and neutral distractor conditions differed significantly with regard to RTs. Although this was to be expected, any difference in the pattern of findings for the two conditions (e.g., stronger distractor repetition benefits for incompatible distractors) might reflect differences in speed rather than differences in the processing of incompatible and neutral distractors. We thus conducted additional analyses to control for this difference. To make RTs for the neutral and incompatible distractor conditions comparable, very slow trials in the incompatible condition (RT $>M+S D$ ) and very fast trials in the neutral condition (RT $<M-$ $S D$ ) were eliminated from the analysis $(M$ and $S D$ were calculated separately within each condition of the repeated measures design for each participant to avoid confounding fast and slow trials with fast vs. slow participants). This procedure completely eliminated the RT difference between the two distractor type conditions (Exp. 1, $F=0.035, p=.97$; Exp. $2, F=0.02, p=.90$ ). We then repeated the same analyses as before with the reduced data set. All relevant results of the standard analyses were completely confirmed: The interaction of distractor repetition and distractor type (incompatible vs. neutral) was significant in both experiments, indicating stronger inhibition for incompatible than for neutral distractors [Exp. 1, $F(1,139)=5.44, p<.05$; Exp. 2, $F(1,81)=5.55, p<$ $.05]$. The interaction of distractor relation and response relation (repetition vs. change) was significant in both experiments, indicating distractorbased response retrieval [Exp. 1, $F(1,139)=293.84, p<.001$; Exp. 2 (without identical stimulus repetitions), $F(1,81)=11.64, p<.01]$. And finally, the three-way interaction remained nonsignificant in both studies $[$ Exp. $1, F(1,139)=2.76$, n.s.; Exp. $2, F(1,81)=2.82$, n.s. $]$.
}

ry processes in a more or less antagonistic manner: Accordingly, inhibition has been assumed to either weaken stimulus-response associations (for a detailed discussion, see Pösse et al., 2006) or to prevent binding processes altogether (Loach et al., 2008; Loach \& Marí-Beffa, 2003). However, those studies employed quite different experimental paradigms (i.e., task switch or rapid serial visual presentation [RSVP]) that constitute particularly demanding selection situations for participants, and thus results of our study and these studies are not easy to compare. Additionally, Tipper et al. (1994) pointed out that as selection becomes more difficult, inhibition might become rather diffuse and could affect a variety of representations of the ignored object instead of only their response properties. A systematic analysis of the relation between inhibition and distractor-response binding in demanding situations is thus an important avenue for further research.

Another topic that deserves further scrutiny in future research regards the exact determinants of the differences in inhibition effects between incompatible and neutral distractors. Research on the so-called "conflict adaptation effect" suggests that encountering a response conflict affects not only the processing of the distracting information in the very trial during which the response conflict occurs, but may also lead to a reactive mobilization of cognitive control processes that affect the selectivity of processing on subsequent trials (Botvinick, Braver, Barch, Carter, \& Cohen, 2001; but see Mayr, Awh, \& Laurey, 2003 , for an alternative explanation of conflict adaptation effects). In the present study, response conflicts either occurred in all trials (incompatible distractor condition) or not at all (neutral distractor condition). We thus cannot decide whether the stronger inhibition of incompatible distractors originated from the specific response conflict that occurred during the prime trial or whether it was due to a generally enhanced level of cognitive control that had its roots in the permanent conflicts that had been encountered during previous trials of the experiment. Disentangling these two components requires a within subject design that allows a separate variation of response conflict during the current and preceding trials. In the present study, we tried to maximize differences in the strength of inhibition by presenting pure sequences of either response-incompatible or neutral trials, which should lead to an accumulation of trial-specific inhibition and global cognitive control processes in the incompatible distractor condition. A major finding of our study was that in spite of the resulting clear differences in the strength of inhibition, processes of distractor-response binding were nevertheless not affected by these differences.

In sum, our findings provide further insight into two processes that are of fundamental importance for the efficient regulation of behavior. Selective attention serves 
the function to prevent irrelevant information from influencing response selection (Allport, 1987; O. Neumann, 1987; Tipper, 1992, 2001). This top-down process of inhibition was shown to operate in a flexible manner, being targeted mainly to those distractors that interfered with response selection (incompatible distractors) rather than to neutral distractors (De Houwer et al., 2001; Frings \& Wentura, 2006; Levinthal \& Lleras, 2008; Tipper et al., 1994). In addition, our findings suggest that inhibition mainly operates by suppressing the link between a distractor and its associated response (Fuentes et al., 1999; Tipper \& Cranston, 1985; Tipper et al., 1994) rather than by deactivating the mental representation of the distractor completely (Houghton \& Tipper, 1994; Houghton et al., 1996).

The binding of stimulus and response information is a second basic mechanism that operates in the service of an automatization of behavior (Denkinger \& Koutstaal, 2009; Hommel, 1998, 2004; Logan, 1988). Re-encountering a familiar stimulus that was present during a previous behavioral episode leads to an automatic retrieval of the previous response, saving cognitive resources for situations that are new and resource-demanding. Apparently, this binding mechanism operates in a non-selective manner, leading to an integration of even those stimuli that are irrelevant for the specification of an action (Frings \& Rothermund, 2011; Frings et al., 2007; Giesen \& Rothermund, 2011; Mayr \& Buchner, 2006; Mayr et al., 2009; Rothermund et al., 2005).

A central implication of our present study is that this bottom-up mechanism of stimulus-driven behavior control is not affected by selective attention, integrating inhibited as well as noninhibited distractors into event files. Such an integration of stimuli and responses might be underlying processes of implicit learning that by definition operate automatically and without requiring attentional resources (e.g., Abrahamse, Jiménez, Verwey, \& Clegg, 2010). In combination with other studies showing that distractorresponse bindings can be modulated by factors like grouping (Frings \& Rothermund, 2011; Möller, Rothermund, \& Frings, 2011) or matching (Giesen \& Rothermund, 2011), our findings support the view that distractor-response binding might be functionally related to feature binding processes in perception (Treisman, 1999), but is functionally independent from processes related to selective attention and response selection.

\section{References}

Abrahamse, E. L., Jiménez, L., Verwey, W. B., \& Clegg, B. A. (2010). Representing serial action and perception. Psychonomic Bulletin \& Review, 17, 603-623.
Akçay, Ç., \& Hazeltine, E. (2007). Conflict monitoring and feature overlap: Two sources of sequential modulations. Psychonomic Bulletin \& Review, 14, 742-748.

Allport, A. (1987). Selection for action: Some behavioral and neurophysiological considerations of attention and action. In $\mathrm{H}$. Heuer \& A. F. Sanders (Eds.), Perspectives on perception and action (pp. 395-419). Hillsdale, NJ: Erlbaum.

Ariga, A., \& Kawahara, J. (2004). The perceptual and cognitive distractor-previewing effect. Journal of Vision, 4, 891-903.

Botvinick, M. M., Braver, T. S., Barch, D. M., Carter, C. S., \& Cohen, J. D. (2001). Conflict monitoring and cognitive control. Psychological Review, 108, 624-652. doi:10.1037/0033-295X.108.3.624

De Houwer, J., Rothermund, K., \& Wentura, D. (2001). Stimulus-feature specific negative priming. Memory \& Cognition, 29, 931-939.

Denkinger, B., \& Koutstaal, W. (2009). Perceive-decide-act, perceivedecide-act: How abstract is repetition-related decision learning? Journal of Experimental Psychology: Learning, Memory, and Cognition, 35, 742-756.

Eriksen, B. A., \& Eriksen, C. W. (1974). Effects of noise letters upon the identification of a target letter in a nonsearch task. Perception \& Psychophysics, 16, 143-149. doi:10.3758/BF03203267

Faul, F., Erdfelder, E., Lang, A.-G., \& Buchner, A. (2007). G*Power 3: A flexible statistical power analysis program for the social, behavioral, and biomedical sciences. Behavior Research Methods, 39, 175191. doi:10.3758/BF03193146

Fletcher, B. C., \& Rabbitt, P. M. (1978). The changing pattern of perceptual analytic strategies and response selection with practice in a two-choice reaction time task. Quarterly Journal of Experimental Psychology, 30, 417-427.

Fox, E. (1994). Interference and negative priming from ignored distractors: The role of selection difficulty. Perception \& Psychophysics, 56, 565-574.

Frings, C., \& Rothermund, K. (2011). To be or not to be... included in an event file: Integration and retrieval of distractors in S-R episodes is influenced by perceptual grouping. Journal of Experimental Psychology: Learning, Memory, and Cognition, 37, 1209-1227.

Frings, C., Rothermund, K., \& Wentura, D. (2007). Distractor repetitions retrieve previous responses to targets. Quarterly Journal of Experimental Psychology, 60, 1367-1377.

Frings, C., \& Wentura, D. (2006). Negative priming is stronger for taskrelevant dimensions: Evidence of flexibility in the selective ignoring of distractor information. Quarterly Journal of Experimental Psychology, 59, 683-693.

Frings, C., \& Wühr, P. (2007). On distractor repetition benefits in the negative-priming paradigm. Visual Cognition, 15, 166-178.

Fuentes, L. J., Vivas, A. B., \& Humphreys, G. W. (1999). Inhibitory tagging of stimulus properties in inhibition of return: Effects on semantic priming and flanker interference. Quarterly Journal of Experimental Psychology, 52A, 149-164.

Gibbons, H., \& Frings, C. (2010). Flanker negative priming from spatially unpredictable primes: An ERP study. Psychophysiology, $75,339-348$.

Gibbons, H., \& Stahl, J. (2008). Early activity in the lateralized readiness potential suggests prime-response retrieval as a source of negative priming. Experimental Psychology, 55, 164-172.

Giesen, C., \& Rothermund, K. (2011). Affective matching moderates S-R binding. Cognition and Emotion, 25, 342-350.

Grison, S., \& Strayer, D. L. (2001). Negative priming and perceptual fluency: More than what meets the eye. Perception \& Psychophysics, 63, 1063-1071. doi:10.3758/BF03194524

Hommel, B. (1998). Event files: Evidence for automatic integration of stimulus-response episodes. Visual Cognition, 5, 183-216. doi: $10.1080 / 713756773$

Hommel, B. (2004). Event files: Feature binding in and across perception and action. Trends in Cognitive Sciences, 8, 494-500. doi:10.1016/j.tics.2004.08.007 
Hommel, B. (2005). How much attention does an event file need? Journal of Experimental Psychology: Human Perception and Performance, 31, 1067-1082.

Hommel, B., \& Colzato, L. (2004). Visual attention and the temporal dynamics of feature integration. Visual Cognition, $11,483-521$

Houghton, G., \& Tipper, S. P. (1994). A model of inhibitory mechanisms in selective attention. In D. Dagenbach \& T. H. Carr (Eds.), Inhibitory processes in attention, memory, and language (pp. 53-112). San Diego, CA: Academic Press.

Houghton, G., Tipper, S. P., Weaver, B., \& Shore, D. I. (1996). Inhibition and interference in selective attention: Some tests of a neural network model. Visual Cognition, 3, 119-164.

Lamy, D., Antebi, C., Aviani, N., \& Carmel, T. (2008). Priming of popout provides reliable measures of target activation and distractor inhibition in selective attention. Vision Research, 48, 30-41.

Levinthal, B. R., \& Lleras, A. (2008). Inter-trial inhibition of attention to features is modulated by task relevance. Journal of Vision, 8, 1-15.

Loach, D., Botella, J., Privado, J., \& Tsotsos, J. K. (2008). Priming and intrusion errors in RSVP streams with two response dimensions. Psychological Research, 72, 281-288.

Loach, D., \& Marí-Beffa, P. (2003). Post-target inhibition: A temporal binding mechanism? Visual Cognition, 10, 513-526.

Logan, G. D. (1988). Toward an instance theory of automatization. Psychological Review, 95, 492-527. doi:10.1037/0033295X.95.4.492

Lorch, E. P., Anderson, D. R., \& Well, A. D. (1984). Effects of irrelevant information on speeded classification tasks: Interference is reduced by habituation. Journal of Experimental Psychology: Human Perception and Performance, 10, 850-864.

MacDonald, P. A., \& Joordens, S. (2000). Investigating a memorybased account of negative priming: Support for selection-feature mismatch. Journal of Experimental Psychology: Human Perception and Performance, 26, 1478-1496.

Mackintosh, B., Mathews, A., \& Holden, E. (2002). Bigger than a breadbox? Attention to distractors may not enhance negative priming. Journal of Experimental Psychology: Human Perception and Performance, 28, 1323-1329.

Mayr, U., Awh, E., \& Laurey, P. (2003). Conflict adaptation effects in the absence of executive control. Nature Neuroscience, 6, 450-452.

Mayr, S., \& Buchner, A. (2006). Evidence for episodic retrieval of inadequate prime responses in auditory negative priming. Journal of Experimental Psychology: Human Perception and Performance, 32, 932-943. doi:10.1037/0096-1523.32.4.932

Mayr, S., Buchner, A., \& Dentale, S. (2009). Prime retrieval of motor responses in negative priming. Journal of Experimental Psychology: Human Perception and Performance, 35, 408-423. doi:10.1037/0096-1523.35.2.408

Mayr, S., Hauke, R., Buchner, A., \& Niedeggen, M. (2008). No evidence for a cue mismatch in negative priming. Quarterly Journal of Experimental Psychology, 62, 645-652. doi:10.1080/ 17470210802483487

Milliken, B., Joordens, S., Merikle, P. M., \& Seiffert, A. E. (1998). Selective attention: A reevaluation of the implications of negative priming. Psychological Review, 105, 203-229.

Möller, B., Rothermund, K., \& Frings, C. (2011). Integrating the irrelevant sound-Grouping modulates the integration of auditory distractors into event files. Manuscript submitted for publication.

Neill, W. T. (1977). Inhibitory and facilitatory processes in selective attention. Journal of Experimental Psychology: Human Perception and Performance, 3, 444-450.

Neill, W. T. (2007). Mechanisms of transfer-inappropriate processing. In D. S. Gorfein \& C. M. MacLeod (Eds.), Inhibition in cognition (pp. 63-78). Washisngton, DC: American Psychological Association.
Neill, W. T., Valdes, L. A., Terry, K. M., \& Gorfein, D. S. (1992). Persistence of negative priming: II. Evidence for episodic trace retrieval. Journal of Experimental Psychology: Learning, Memory, and Cognition, 18, 993-1000.

Neumann, O. (1987). Beyond capacity: A functional view of attention. In H. Heuer \& A. F. Sanders (Eds.), Perspectives on perception and action (pp. 361-394). Hillsdale, NJ: Erlbaum.

Neumann, E., \& DeSchepper, B. G. (1991). Costs and benefits of target activation and distractor inhibition in selective attention. Journal of Experimental Psychology: Learning, Memory, and Cognition, 17, 1136-1145.

Park, J., \& Kanwisher, N. (1994). Negative priming for spatial locations: Identity mismatching, not distractor inhibition. Journal of Experimental Psychology: Human Perception and Performance, 20, 613-623.

Pashler, H., \& Baylis, G.C. (1991). Procedural learning: II. Intertrial repetition effects in speeded-choice tasks. Journal of Experimental Psychology: Learning, Memory, and Cognition, 17, 33-48.

Pösse, B., Waszak, F., \& Hommel, B. (2006). Do stimulus-response bindings survive a task switch? European Journal of Cognitive Psychology, 18, 640-651.

Rothermund, K., Wentura, D., \& De Houwer, J. (2005). Retrieval of incidental stimulus-response associations as a source of negative priming. Journal of Experimental Psychology: Learning, Memory, and Cognition, 31, 482-495. doi:10.1037/02787393.31.3.482

Stablum, F., Ricci, R., Pavese, A., \& Umiltà, C. (2001). Global/local processing and negative priming: The influence of selection difficulty and stimulus exposure. Psychological Research, 65, 81-97.

Tipper, S. P. (1985). The negative priming effect: Inhibitory priming by ignored objects. Quarterly Journal of Experimental Psychology, 37, 571-590.

Tipper, S. P. (1992). Selection for action: The role of inhibitory mechanisms. Current Directions in Psychological Science, 1, $105-109$.

Tipper, S. P. (2001). Does negative priming reflect inhibitory mechanisms? A review and integration of conflicting views. Quarterly Journal of Experimental Psychology, 54, 321-343.

Tipper, S. P., \& Cranston, M. (1985). Selective attention and priming: Inhibitory and facilitatory effects of ignored primes. Quarterly Journal of Experimental Psychology, 37, 591-611.

Tipper, S. P., Weaver, B., \& Houghton, G. (1994). Behavioural goals determine inhibitory mechanisms of selective attention. Quarterly Journal of Experimental Psychology, 47A, 809-840.

Treisman, A. (1999). Feature binding, attention and object perception. In G. W. Humphreys, J. Duncan, \& A. Treisman (Eds.), Attention, space and action: Studies in cognitive neuroscience (pp. 91-111). Oxford: Oxford University Press.

Tukey, J. W. (1977). Exploratory data analysis. Reading, MA: Addison-Wesley.

Wühr, P., \& Müsseler, J. (2005). When do irrelevant visual stimuli impair processing of identical targets? Perception \& Psychophysics, 67, 897-909.

Yashar, A., \& Lamy, D. (2010). Intertrial repetition facilitates selection in time: Common mechanisms underlie spatial and temporal search. Psychological Science, 21, 243-251. doi:10.1177/ 0956797609357928

\section{Author notes}

The research reported in this article was supported by a grant of the Deutsche Forschungsgemeinschaft to K.R. and C.F. (DFG RO 1272/6-1). 